\title{
Comparitive Study Between Early Menopause and Premenopausal Rheumatoid Arthritis patients
}

\author{
Nesma M. Mohhmed, Mohamed A. Esmail and Osama S. DaifAllah \\ Department of Rheumatology and Rehab ilitation, Sohag Faculty of Medicine, Sohag \\ University
}

\begin{abstract}
Introduction: Rheumatoid arthritis (RA) is a chronic autoimmune systemic inflammatory disease marked by symmetric, peripheral polyarthritis. It is the most common form of inflammatory arthritis and often results in joint damage and physical disability. Exposure to estrogen may be protective against the onset of disease, yet earlier age at menopause may be associated with a greater proportion of patients presenting with a milder disease course

Aim of the work: Assess the effect of early menopause on the activity and severity of rheumatoid arthritis.

Patients and Methods: Cross sectional clinical study, included 50 patient divided on 2 groups (premenopausal and early menopausal), diagnosed as RA based on ACR/EULAR classification criteria 2010.

Results: Disease activity was higher in earlymenopause group than premenopause group.

Conclusion: Early menopause is associated with more active and sever type of RA among women with disease onset after 45 years of age.
\end{abstract}

Key words: RA, early menopause, premenopause.

\section{Introduction}

Rheumatoid arthritis (RA) is a chronic autoimmune systemic inflammatory disease marked by symmetric, peripheral polyarthritis. It is the most common form of inflammatory arthritis and often results in joint damage and physical disability. As it is systemic disease, it may result in a variety of extra-articular manifestations, including fatigue, subcutaneous nodules, lung involvement, pericarditis, peripheral neuropathy, vasculitis and hematologic abnormalities $^{(\mathbf{1})}$.

Menopause occurs at an average age of 51 years and marks a period of significant hormonal change for women, impacting a range of organs and body systems, including the cardiovascular and musculoskeletal system $^{(\mathbf{1})}$.

Interactions between levels of sex hormones (estrogen, progesterone) and the immune system are complex, but decreases in hormone production, as occurs during menopause, can be associated with an increase in disease activity in RA. Indeed, early menopause may be a factor that increases risk for developing RA and the post-menopausal period is associated with the most significant levels of joint damage and disability in women with RA ${ }^{(\mathbf{1})}$.

Hormonal factors have been suggested to influence the risk of RA.A reduced incidence occurs during pregnancy, and also an increased rate of clinical remission among women with RA who become pregnant ${ }^{(2)}$.

Low testosterone levels have been described in both women and men with $\mathrm{RA}^{(3)}$ and a high incidence of RA is found during the peri- and postmenopausal period in women, suggesting that hormones and hormonal changes may influence pathogenesis and disease progression ${ }^{(4)}$.

These suggest that exposure to estrogen may be protective against the onset of disease, yet earlier age at menopause may be associated with a 
greater proportion of patients presenting with a milder disease course ${ }^{(\mathbf{4})}$.

\section{Aim of the work:}

1. Assess the effect of early menopause on the activity and severity of rheumatoid arthritis.

2. Investigate whether hormonal factors such as breastfeeding history, oral contraceptive use, and menopausal age, influence severity of RA.

\section{Patients and Methods:}

Design: Cross sectional clinical study. Patients:

This study included 50 patient divided on 2 groups, all studied patients were patients with rheumatoid arthritis based on clinical and laboratory criteria of the American college of rheumatology ACR/EULAR RA classification criteria 2010.

1. The first group consist of 25 cases had early menopause that happened at age 45 years or younger.

2. The second group is 25 cases were premenopausal.

All patients subjected to clinical evaluation and we stressed on musculoskeletal examination.

\section{Methods:}

All patients interviewed for demographic data (age, sex, occupation, age at onset of the disease, joints affected,extra-articular features, Rheumatoid factor, family history, treatment regimen. We assessed Medical and rheumatological history.

Careful general, musculoskeletal examination was done.

Assessment of disease activity performed using the Modified Disease Activity Score (MDAS28) including 28 tender and swollen joint count, ESR, visual analogue score. DAS28 score of higher than 5.1 is indicative of high disease activity, whereas a DAS28 below 3.2 indicates low disease activity. A patient is considered to be in remission if they have a DAS28 lower than 2.6. Disease severity was assessed by radiological scoring (Larsen score, hand X-ray AP view).

Health related quality of life measured by modified health assessment questionnaire (M-HAQ). Ask the patient to complete the entire questionnaire by circling the appropriate responses while in the waiting room prior to his/her visit. Add the totals from each of the four columns to get the patient's MHAQ score. Divide this score by 8-your result should fall between 0.0 and 3.0. The higher the score, the greater the overall disability. MHAQ scores $<0.3$ are considered normal, although the average MHAQ in the general population increases with age.

Data on reproductive factors, such as OC use (including number of years taken and when started), number of births, whether each child was breast-fed and for how many months, age at menarche and if applicable age at menopause was obtained using a self-administered questionnaire. OC use was stratified into never used, used OCS. Breast-feeding history was stratified into never breast-fed, breast fed all her children. Early menarche was defined as menarche before the age of twelve, and early menopause as reported menopause at the age of 45 or younger.

Laboratory investigations done to all patients (CBC, ESR, RF, RFTS, LFTS, urine analysis)

Clinical outcomes, including erosions on X-rays, RF status, use of DMARDs and disability measured with MHAQ, DAS 28 score, Larsen score were collected and we compared between 2 groups regarding indicators of disease activity and severity.

\section{Inclusion criteria:}

1) Patients with RA classified according to the revised American College of Rheumatology (ACR)/ EULAR RA classification criteria 


\section{0 for RA.}

2) Age $>18$ years.

3) Patients who are able and willing to give written informed consent.

\section{Exclusion criteria:}

1) Any other collagen disease rather than RA.

2) Young patient $<18$ years.

3) Patients not able and willing to give written informed consent.

\section{Results}

The mean age of the studied was 52.3 among early menopausal patients, compared to 35.6 among premenopausal patients, with significant difference (Table $1)$.

Table 1. Age of the studied groups

\begin{tabular}{|l|l|c|c|c|}
\hline & Group & Mean & Std. Deviation & Std. Error Mean \\
\hline \multirow{2}{*}{ Age } & Premenopause & 35.60 & 5.545 & 1.109 \\
\cline { 2 - 5 } & Early menopause & 52.28 & 5.734 & 1.147 \\
\hline
\end{tabular}

$\mathrm{T}$ test $=10.456, \mathrm{p}$ value $<\mathbf{0 . 0 0 1}(\mathrm{HS})$

The mean duration since menopause in early menopause group was 8.24 years, with a relatively high standard deviation (5.6 years). This was reflected in the very wide range (from 1-17 years). More than $80 \%$ of cases were married, and there was non significant difference between the two groups (Table 2).

Table 2. Marital state of the studied groups

\begin{tabular}{|c|c|c|c|c|c|}
\hline & \multicolumn{2}{|l|}{ group } & \multirow[t]{2}{*}{ Total } \\
\hline & & & $\begin{array}{l}\text { Premenopaus } \\
\mathrm{e}\end{array}$ & Early menopause & \\
\hline \multirow{16}{*}{$\begin{array}{l}\text { marital } \\
\text { status }\end{array}$} & \multirow[t]{4}{*}{ Single } & Count & 3 & 2 & 5 \\
\hline & & $\%$ within marital status & $60.0 \%$ & $40.0 \%$ & $100.0 \%$ \\
\hline & & $\%$ within group & $12.0 \%$ & $8.0 \%$ & $10.0 \%$ \\
\hline & & $\%$ of Total & $6.0 \%$ & $4.0 \%$ & $10.0 \%$ \\
\hline & \multirow{4}{*}{$\begin{array}{l}\text { Marrie } \\
\text { d }\end{array}$} & Count & 22 & 20 & 42 \\
\hline & & $\%$ within marital status & $52.4 \%$ & $47.6 \%$ & $100.0 \%$ \\
\hline & & $\%$ within group & $88.0 \%$ & $80.0 \%$ & $84.0 \%$ \\
\hline & & $\%$ of Total & $44.0 \%$ & $40.0 \%$ & $84.0 \%$ \\
\hline & \multirow{4}{*}{$\begin{array}{l}\text { Divorc } \\
\text { ed }\end{array}$} & Count & 0 & 1 & 1 \\
\hline & & $\%$ within marital status & $0.0 \%$ & $100.0 \%$ & $100.0 \%$ \\
\hline & & $\%$ within group & $0.0 \%$ & $4.0 \%$ & $2.0 \%$ \\
\hline & & $\%$ of Total & $0.0 \%$ & $2.0 \%$ & $2.0 \%$ \\
\hline & \multirow[t]{4}{*}{ Widow } & Count & 0 & 2 & 2 \\
\hline & & $\%$ within marital status & $0.0 \%$ & $100.0 \%$ & $100.0 \%$ \\
\hline & & $\%$ within group & $0.0 \%$ & $8.0 \%$ & $4.0 \%$ \\
\hline & & $\%$ of Total & $0.0 \%$ & $4.0 \%$ & $4.0 \%$ \\
\hline \multirow{4}{*}{\multicolumn{2}{|c|}{ Total }} & Count & 25 & 25 & 50 \\
\hline & & $\%$ within marital status & $50.0 \%$ & $50.0 \%$ & $100.0 \%$ \\
\hline & & $\%$ within group & $100.0 \%$ & $100.0 \%$ & $100.0 \%$ \\
\hline & & $\%$ of Total & $50.0 \%$ & $50.0 \%$ & $100.0 \%$ \\
\hline
\end{tabular}

The duration of disease was longer in menopause group than premenopause group (Table 3 ). 
Table 3. Duration of disease

\begin{tabular}{|l|l|l|l|l|}
\hline & Group & N & Mean & $\begin{array}{l}\text { Std. } \\
\text { Deviation }\end{array}$ \\
\hline $\begin{array}{l}\text { duration of disease } \\
\text { in years }\end{array}$ & Premenopause & 25 & 6.080 & 4.8663 \\
\cline { 2 - 5 } & Early menopause & 25 & 13.280 & 7.4918 \\
\hline
\end{tabular}

Only 3 patients had family history ( 2 from the premenupause group and one from the early menupause group). The difference between the two groups was non significant, also in premenupause group, about half of cases had gradual onset, and another third had insidious onset, with the last 4 cases had acute onset. In the early menopause group, the majority of cases had insidious onset $(62.5 \%)$, with the remaining cases had gradual onset, and non had acute onset. The difference between the two groups was significant.

Patients in the premenopause group had either no morning stiffness (13 cases) or morning stiffness of less than one hour (12 cases). This was completely different from patients of the early menopause group as all of them had morning stiffness of different durations, and 19 of the 25 cases $(76 \%)$ had morning stiffness of more than one hour (Table 4).

Table 4. Morning stiffness in the studied groups

\begin{tabular}{|c|c|c|c|c|c|}
\hline & \multicolumn{2}{|l|}{ group } & \multirow[t]{2}{*}{ Total } \\
\hline & & & Premenopause & $\begin{array}{l}\text { Early } \\
\text { menopa } \\
\text { use }\end{array}$ & \\
\hline \multirow{12}{*}{$\begin{array}{l}\text { Morning } \\
\text { stiffness }\end{array}$} & \multirow[t]{3}{*}{ No } & Count & 13 & 0 & 13 \\
\hline & & $\%$ within Morning stiffness & $100.0 \%$ & $0.0 \%$ & $\begin{array}{l}100 \\
\%\end{array}$ \\
\hline & & $\%$ within group & $52.0 \%$ & $0.0 \%$ & $\begin{array}{l}26.0 \\
\%\end{array}$ \\
\hline & \multirow[t]{3}{*}{$<30$ min } & Count & 8 & 1 & 9 \\
\hline & & $\%$ within Morning stiffness & $88.9 \%$ & $11.1 \%$ & $100 \%$ \\
\hline & & $\%$ within group & $32.0 \%$ & $4.0 \%$ & $\begin{array}{l}18.0 \\
\%\end{array}$ \\
\hline & \multirow[t]{3}{*}{$30 \mathrm{~min}-1 \mathrm{hr}$} & Count & 4 & 5 & 9 \\
\hline & & $\%$ within Morning stiffness & $44.4 \%$ & $55.6 \%$ & $100 \%$ \\
\hline & & $\%$ within group & $16.0 \%$ & $20.0 \%$ & $\begin{array}{l}18.0 \\
\%\end{array}$ \\
\hline & \multirow[t]{3}{*}{$>1 \mathrm{hr}$} & Count & 0 & 19 & 19 \\
\hline & & $\%$ within Morning stiffness & $0.0 \%$ & $100.0 \%$ & $100 \%$ \\
\hline & & $\%$ within group & $0.0 \%$ & $76.0 \%$ & $\begin{array}{l}38.0 \\
\%\end{array}$ \\
\hline \multirow{3}{*}{\multicolumn{2}{|c|}{ Total }} & Count & 25 & 25 & 50 \\
\hline & & $\%$ within Morning stiffness & $50.0 \%$ & $50.0 \%$ & $100 \%$ \\
\hline & & $\%$ within group & $100.0 \%$ & $100.0 \%$ & $100 \%$ \\
\hline
\end{tabular}

Mean of age at menarche about 12 years in both groups and this difference was non significant. Number of patients who used COCs is more in premenopause group. Mean of no of pregnancies was more or less similar in the 2 groups and difference was non significant. One patient has D.M (from premenopause group), no patients has D.M from another group and this difference was non significant. 3 patients has HTN (2 from premenopause group, 1 from early menopause group) and this difference was 
non significant. Most of cases had history of breast feeding (over 80\%) and the number of patients who had history of breastfeeding was equal in both groups and this was non significant.

Table 5 show that number of swollen\&tender joints in early menopause group was more than premenopause group, also scores of disease activity (DAS 28, Larsen score, MHAQ) were higher in early menopause group than premenopause group.

Table 5. Comparison between the two groups regarding disease activity

\begin{tabular}{|l|l|l|l|}
\hline & Premenopausal & Earlymenopausal & P value \\
\hline No of swollen joints & $1 \pm 1.08$ & $3.68 \pm 2.50$ & $<0.001$ \\
\hline No of tender joints & $1.92 \pm 1.32$ & $5 \pm 2.06$ & $<0.001$ \\
\hline DAS28 & $3.63 \pm 0.63$ & $5.07 \pm 0.42$ & $<0.001$ \\
\hline Larsen score & $5.76 \pm 12.93$ & $40.84 \pm 25.52$ & $<0.001$ \\
\hline MHAQ & $0.1840 \pm 0.3032$ & $1.1950 \pm 0.4974$ & $<0.001$ \\
\hline
\end{tabular}

Table 6 show that only 2 patients had skin manifestations (from early menopause group), 5 patients had dry eye (from early menopause group), no patients had extraarticular manifestations from premenopause group. All premenopause patients had no deformities but 7 patients from early menopause group had different deformities and difference was significant.

Table 6. Extraarticular manifestations of the two groups

\begin{tabular}{|l|l|l|l|l|}
\hline \multicolumn{2}{|l|}{ Variable } & Premenopause & $\begin{array}{l}\text { Early } \\
\text { menopause }\end{array}$ & \multirow{2}{*}{ P value } \\
\hline \multirow{3}{*}{ Skin } & No & $25(100 \%)$ & $23(92 \%)$ & \multirow{2}{*}{$0.240(\mathrm{NS})$} \\
\cline { 2 - 4 } & Raynaud's & 0 & $1(4 \%)$ & \\
\cline { 2 - 4 } & Rh nodule & 0 & $1(4 \%)$ & \\
\hline Eye & No & $25(100 \%)$ & $20(80 \%)$ & \multirow{2}{*}{$0.05(\mathrm{~S})$} \\
\hline & Dry eye & 0 & $5(20 \%)$ & \\
\hline Pulmonary & No & $25(100 \%)$ & $25(100 \%)$ & - \\
\hline Neurological & No & $25(100 \%)$ & $25(100 \%)$ & - \\
\hline Haematological & No & $25(100 \%)$ & $25(100 \%)$ & - \\
\hline Cardiac & No & $25(100 \%)$ & $25(100 \%)$ & - \\
\hline GIT & No & $25(100 \%)$ & $25(100 \%)$ & - \\
\hline Renal & No & $25(100 \%)$ & $25(100 \%)$ & - \\
\hline Hepatic & No & $25(100 \%)$ & $25(100 \%)$ & - \\
\hline
\end{tabular}

Only 19 patients were anemic ( 9 patients from premenopause group and 10 from early menopause group) and this difference was non significant. Patients who had high titre RF were more in early menopause group than premenopause group and this difference was significant $(\mathrm{p}$ value $=0.008$ ), also number of patients that had abnormal ESR was more in early menopause group than premenopausal patients.

As regards treatment, premenopause patients take MTX in doses lower than those taken by early menopause patients, and this difference was significant. Only 10 patients not take NSAIDS (4 from premenopause group and 6 from Early menopause group) and difference was non significant. The number of patients and doses of other DMARDs than MTX was similar between the two groups with non significant differences. Mean of corticosteroid dose was similar in both groups with non significant difference. 


\section{Discussion}

In this study, women with a history of early menopause who developed RA were more likely to have active and sever disease course. This suggests that different predictors may be associated with distinct clinical outcomes. For example, other known risk factors for RA, such as the shared epitope of HLA-DRB1, and smoking, may, in comparison with early menopause, be more specifically associated with a severe phenotype of $\mathrm{RA}^{(5)}$.

This study consisted of 50 patients divided on 2 groups (early menopause and premenopause group), each group consisted of 25 patient. Mean age of early menopause study group was 52 year with standard deviation 5.7 and in premenopause study group mean age was 35 with standard deviation 5.5. This was different from study done by Karlson et al. ${ }^{(5)}$ as mean age of their patients was 63 years, with a range of 47 to 80 years. Majority of our cases were in the poor socioeconomic class, and this was fixed in both groups, also $80 \%$ of cases were married.

The mean age at menopause in second group in our study was 44 year with standard deviation (0.7489) and range from 43 to 45 . This was different from study done by Merlino et al. ${ }^{(6)}$ as mean age at menopause in their study was 47 year with standard deviation (0.6) and range from 45 to 57.

In this study mean of duration of disease was in premenopause group 6 years with standard deviation 4.8 and in early menopause group was 13 year with standard deviation (7.4). Pikwer et al. ${ }^{(7)}$ also reported in their study that mean disease duration at the last follow-up was 10.1 years (SD, 3.5). This was different from study made by
Karlson et al. ${ }^{(5)}$ as mean of disease duration was 9 years.

In our study it was found that patients in the premenopause group had either no morning stiffness (13 cases) or morning stiffness of less than one hour (12 cases). This was completely different from patients of the early menopause group as all of them had morning stiffness of different durations, and 19 of the 25 cases $(76 \%)$ had morning stiffness of more than one hour.

This mean that disease was more active in early menopause patients than premenopause patients that confirm effect of estrogen hormone on RA. Estrogen is suggested to supress cellular immunity and stimulates a shift towards Thelper 2 subset. An early drop of estrogen levels, i.e. early menopause, may therefore enhance the risk of RA, since Th1 cells have been implicated to be an important part of the pathogenesis of RA. There are other prior studies that have found similar results about the effect of menopausal age on the risk of developing RA. Merlino et al. (6) reported that women who reached menopause at the age of 51 had a reduced risk of RA.

It was found that mean of age at menarche about 12 years in 2 groups. This was different from study made by Karlson et al. ${ }^{(5)}$ as age at menarche was $<12$ year. In study of Pikwer et al. (7) they found an inverse association between early menarche and RA.

In our study it was found that most of cases had history of breast feeding (over $80 \%$ ) and the number of patients who had history of breastfeeding was equal in both groups. This is different from study made by Barrett et al. ${ }^{(9)}$ who suggested that breastfeeding is a risk factor for a more-severe inflammatory polyarthritis 
in a small group of genetically susceptible women, based on the linkage between HLA-DRB1 alleles and the prolactin gene on chromosome $6^{(8)}$.

In a prospective short-term study of pregnant women with inflammatory polyarthritis, they found that women who breastfed after a first pregnancy had a more-severe disease 6 months postpartum compared with nonbreastfeeders and previous breastfeeders ${ }^{(9)}$. However, in the present study, we could not demonstrate any significant long-term effect of breastfeeding on the severity of RA.

It was found that number of patients who used COCS is more in premenopause group than early menopause group. This was similar study to done by Jorgensen et al. ${ }^{(10)}$ found that $\mathrm{OC}$ is both protective for RA and associated with a mild phenotype in individuals who develop RA.

In study of Camacho et al. ${ }^{(11)}$ it was suggested that $\mathrm{OC}$ use is associated with mild RA, and demonstrated a reduction of disability, measured by HAQ, over time in former OC users compared with nonusers. Furthermore, current OC users had lower HAQ scores at baseline and over time than previous users. This was different from that reported by Pikwer et al. ${ }^{(7)}$ as they did not see any significant effect of OC use, or stressrelated factors, on the risk of RA, in their study there was not any strong trend with OC-use observed, but there was a long lag period between OC-use and RA-development in their sample.

In this study it was found that number of swollen \& tender joints is more in early menopause patients than premenopause patients. Mean of all Larsen score, MHAQ and DAS 28 higher in early menopause group higher than premenopause group, also all premenopause patients had no deformities but 7 patients from early menopause group had different deformities. This is confirm finding of studies done by Pikwer et al. (7), Camacho et al. (11) who found that mean of MHAQ score was (0.9) higher in early menopause patients with range from $(0.7-1.1)$.

These results indicate that hormones are involved in the complex pathogenesis of RA. Possible explanations for the protective effect of breast-feeding, OC use could be long term immunomodulation, a dysregulated HPA axis or unmeasured confounders. Although the association remained significant in a model adjusted for smoking and level of formal education, we cannot exclude that other life style factors or exposures associated with long term breastfeeding could play a role ${ }^{(\mathbf{1 1})}$.

\section{Conclusion:}

We report that early menopause is associated with more active type of RA among women with disease onset after 45 years of age. Hormonal changes may influence pathways that are distinct from those leading to severe, progressive disease. To our knowledge, this is the first study on the impact of menopausal age on the severity of RA, and our findings must be confirmed in other studies of larger samples. Such studies require that data on the exposure (that is, age at menopause) have been collected in a consistent and valid manner. If our results are confirmed, it may in the future be relevant to check for early menopause in women with RA.

\section{References:}

1. Sammaritano LR. Menopause in patients with autoimmune diseases. Autoimmunity reviews. 2012;11(67):A430-6. 
2. Hazes JM, Coulie PG, Geenen V, Vermeire S, Carbonnel F, Louis E, et al. Rheumatoid arthritis and pregnancy: evolution of disease activity and pathophysiological considerations for drug use. Rheumatology. 2011;50(11):1955-68.

3. Tengstrand B, Carlstrom $\mathrm{K}$, Hafstrom I. Gonadal hormones in men with rheumatoid arthritis-from onset through 2 years. The Journal of rheumatology. 2009;36(5):887-92.

4. Doran MF, Pond GR, Crowson CS, O'Fallon WM, Gabriel SE. Trends in incidence and mortality in rheumatoid arthritis in Rochester, Minnesota, over a forty-year period. Arthritis and rheumatism. 2002;46(3):625-31.

5. Karlson EW, Mandl LA, Hankinson SE, Grodstein F. Do breast-feeding and other reproductive factors influence future risk of rheumatoid arthritis? Results from the Nurses' Health Study. Arthritis and rheumatism. 2004;50(11):3458-67.

6. Merlino LA, Cerhan JR, Criswell LA, Mikuls TR, Saag KG. Estrogen and other female reproductive risk factors are not strongly associated with the development of rheumatoid arthritis in elderly women.
Seminars in arthritis and rheumatism. 2003;33(2):72-82.

7. Pikwer $M$, Bergstrom U, Nilsson JA, Jacobsson L, Turesson C .Early menopause is an independent predictor of rheumatoid arthritis. Annals of the rheumatic diseases. 2012;71(3):378-81.

8. Brennan P, Ollier B, Worthington J, Hajeer A, Silman A. Are both genetic and reproductive associations with rheumatoid arthritis linked to prolactin? Lancet. 1996;348(9020):106-9.

9. Barrett JH, Brennan P, Fiddler M, Silman A. Breast-feeding and postpartum relapse in women with rheumatoid and inflammatory arthritis. Arthritis and rheumatism. 2000;43(5):1010-5.

10. Jorgensen C, Picot MC, Bologna C, Sany J. Oral contraception, parity, breast feeding, and severity of rheumatoid arthritis. Annals of the rheumatic diseases. 1996;55(2):94-8.

11. Camacho EM, Lunt M, Farragher TM, Verstappen SM, Bunn DK, Symmons DP. The relationship between oral contraceptive use and functional outcome in women with recent-onset inflammatory polyarthritis: results from the Norfolk Arthritis Register. Arthritis and rheumatism. 2011;63(8):2183-91. 